\title{
Visualización Matemática con GEOGEBRA: Integrales Impropias
}

\author{
Miguel Delgado Pineda \& Adoración Medina Albós \\ Grupo de Innovación en Matemáticas. $\boldsymbol{\pi}$-Mat \\ Departamento de Matemática Fundamentales, \\ Universidad Nacional De Educación a Distancia, UNED \\ C/ Senda del Rey 9 (28040 Madrid), \\ e-mail: miguel@mat.uned.es \& marmedina@madrid.uned.es \\ Presentado en eXIDO17 (2017)
}

\section{RESUMEN}

Este trabajo presentamos un proyecto de innovación docente que utiliza el software Geogebra de Geometría Dinámica, con el objeto facilitar el aprendizaje de las integrales impropias a los estudiantes de primer curso del Grado de Ciencias Matemáticas en la UNED. Para ello se ha desarrollado una ingeniería visual, que se basa en la utilización de dos laboratorios de simulación matemática. Estos laboratorios van más allá de la simple representación dinámica de problemas y conceptos. Están específicamente diseñados para incidir en el proceso de Visualización Matemática de los estudiantes, entendiéndose este como el proceso de codificación de información matemática en una imagen. Este proyecto ha sido desarrollado en el marco de las acciones del Grupo de Innovación en Matemáticas $\pi$-Mat.

Palabras clave: Innovación Docente, Visualización Matemática, Laboratorios de Simulación, Educación Matemática, GeoGebra.

\section{INTRODUCCION}

El presente proyecto tiene como objetivo el desarrollo y utilización de un laboratorio de simulación matemática para favorecer los procesos de visualización matemáticas en los estudiantes de Análisis Matemático de primer curso del Grado de Matemáticas. En el laboratorio se dispone de dos opciones; una relativa al primer criterio de comparación para integrales impropias y otra relativo al segundo criterio de comparación. Tanto para integrales impropias de primera especie como de segunda especie. El laboratorio está diseñados para que el estudiante experimente y estudie con distintas funciones integrando. Está dirigido a los estudiantes que cursan la asignatura Funciones de una variable II, puesto que es en esta asignatura donde se aborda el estudio de las integrales impropias. 
Comunicaciones sobre innovación en la enseñanza y el aprendizaje del Análisis Matemático

Este laboratorio es una pequeña parte de un proyecto amplio para facilitar todos los contenidos de las asignaturas Funciones de una variable I y Funciones de una variable II. Los descriptores de los contenidos son:

\begin{tabular}{|l|l|}
\hline Funciones de una variable I & Funciones de una variable II \\
\hline Sucesiones & Integral de Riemann \\
\hline Los números reales & Teoremas Fundamentales del Cálculo \\
\hline Límites infinitos & $\begin{array}{l}\text { Funciones Logarítmicas } \\
\text { Exponenciales }\end{array}$ \\
\hline Topología en R & Funciones Trigonométricas \\
\hline Límites de funciones & Integrales Impropias \\
\hline Funciones continuas & Integrales Eulerianas \\
\hline Funciones derivables & Sucesiones de Funciones \\
\hline Funciones derivables en intervalos & Series de Funciones \\
\hline El Teorema de Taylor & Series de Potencias \\
\hline $\begin{array}{l}\text { Límites superior e inferior de una } \\
\text { sucesión de números reales }\end{array}$ & \\
\hline Series de números reales & \\
\hline
\end{tabular}

\section{Los Laboratorios de Simulación Matemática}

Nuestro laboratorio se han desarrollado como una pila de objetos e instrucciones de GeoGebra con un marcado potencial experimental, tanto en numérico como en abstracto, para uso didáctico para facilitar tanto la enseñanza como el aprendizaje de las matemáticas. En esta aplicación, GeoGebra, se combina herramientas de álgebra, geometría, análisis matemático y estadística con muy buenas prestaciones gráficas y geométricas. En nuestro laboratorio utilizamos, principalmente, el marco gráfico (Vista Gráfica) y el marco algebraico (Vista Algebraica). Estas vistas interactúan de forma dinámica entre si, de modo que cualquier expresión algebraica introducida en la vista algebraica se representa en la vista gráfica y cualquier objeto representado en la vista gráfica con las diferentes herramientas de las que dispone el programa aparece representado algebraicamente en la vista algebraica.

En la hoja GeoGebra del laboratorio se definen objetos sobre los cuales se modifican sus contenidos y sus propiedades, de forma que el estudiante interactúa con unos menús sin necesidad de ser un estudioso de la herramienta. Es decir, los laboratorios son objetos dinámicos sobre los cuales el profesor ejerce un control contra usos que no desea. El estudiante tan sólo puede interactuar con los objetos contenidos en la hoja, y no puede salirse de este 
Comunicaciones sobre innovación en la enseñanza y el aprendizaje del Análisis Matemático

conjunto de objetos. Además, estos los laboratorios son accesibles vía internet sin necesidad de instalar la aplicación en sus ordenadores.

Como presentar todos los laboratorios que hemos diseñado (y que seguimos diseñando) sería demasiado ambicioso para este artículo, presentamos con todo detalle dos de los mismos.

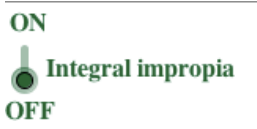

Laboratorio de Simulación Matemática
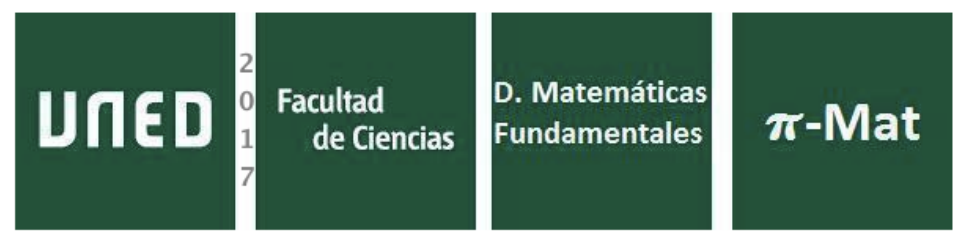

Miguel Delgado Pineda \& Adoración Medina Albós

Figura 1: Imagen inicial del laboratorio.

Los matemáticos experimentables son los criterios de comparación que describimos para integrales impropias de primera especie

Primer Criterio de Comparación: Sean f y g dos funciones de $[a,+\infty)$ a $\mathbb{R}$ que son integrables en el intervalo $[a, \alpha], \forall \alpha \geq a$, tales que existe un número $b>a$ y $0 \leq g(x) \leq f(x) \forall x \geq b$. Si $\int_{a}^{+\infty} f(x) d x$ es convergente (divergente), entonces la $\int_{a}^{+\infty} g(x) d x$ es convergente (divergente).

Segundo Criterio de Comparación: Sean fy g dos funciones de $[a,+\infty)$ a $\mathbb{R}$ que son integrables en el intervalo $[a, \alpha], \forall \alpha \geq a$, tales que

$$
\lim _{x \rightarrow+\infty} \frac{g(x)}{f(x)}=L \in \mathbb{R}
$$

- Si $L \neq 0$, entonces $\int_{a}^{+\infty} f(x) d x$ y $\int_{a}^{+\infty} g(x) d x$ tienen el mismo carácter.

- Si $L=0$ y $\int_{a}^{+\infty} f(x) d x$ es convergente, entonces $\int_{a}^{+\infty} g(x) d x$ es convergente.

Para poder utilizar alguno de los dos criterios es necesario disponer de una función $f(x)$ de cuya integral impropia sea conocido su carácter. Nuestras funciones muestra para la comparación son

$$
f(x)=\frac{1}{\left(x-x_{0}\right)^{p}} \quad \text { con } \quad p>0 .
$$


Comunicaciones sobre innovación en la enseñanza y el aprendizaje del Análisis Matemático

Esto es así puesto que la integral impropia

$$
\int_{a}^{+\infty} \frac{d x}{\left(x-x_{0}\right)^{p}} \quad \text { con } \quad a>x_{0} .
$$

que converge para cada $p>1$ y divergen si $p \leq 1$.

\section{Experimentación con el laboratorio}

El estudiante puede cambiar de función de comparación eligiendo en cualquier momento el valor del parámetro $p$ para poder experimentar con distintas funciones que se adecuen a la función de estudio $g(x)$.

Este función de comparación mediante un deslizador varía el valores del exponente de

y otro deslizador varía el valor de con $x_{0}$.

$$
f(x)=\frac{1}{\left(x-x_{0}\right)^{p}}
$$

La función integrando $g(x)$ de la integral impropia que se quiere estudiar, $\int_{a}^{+\infty} g(x) d x$, es introducida por el estudiante mediante una casilla de entrada en la que se escribe la expresión del integrando.

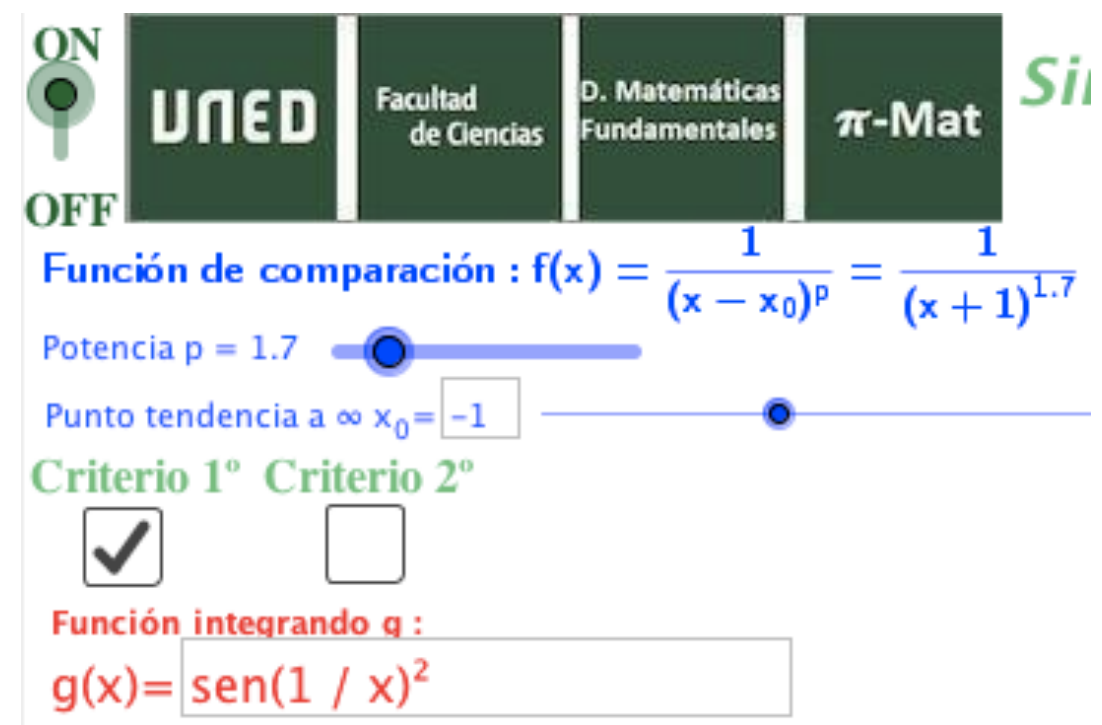

Figura 2: Función integrando y función de comparación.

Una vez definida el integrando $g(x)$ puede optarse por ver una representación gráfica de esa función y de la función de comparación. Al hacerlas visibles se aprecian las opciones elegibles correspondientes a las dos integrales impropias. 
En cualquier momento de proceso puede cambiar la elección de criterio, bien el primero o bien el segundo. Con la selección del segundo se activa la elección gráfica de la función cociente $\frac{g}{f}$.

Si la integral es de primera especie, $\int_{a}^{+\infty} g(x) d x$, el objetivo principal es saber el carácter de la integral, puesto que en aquellas ocasiones en las cuales la función integrando posee función primitiva la opción determina el valor de dicha integral.

La integral impropia a parece como texto y como zona gráfica a la vez, de manera que puedan compararse dichas zonas gráficas tanto numéricamente como visualmente.

En general, la integral impropia $\int_{a}^{+\infty} f(x) d x$ es valorado sin dificultad pues es conocida una función primitiva del integrando.

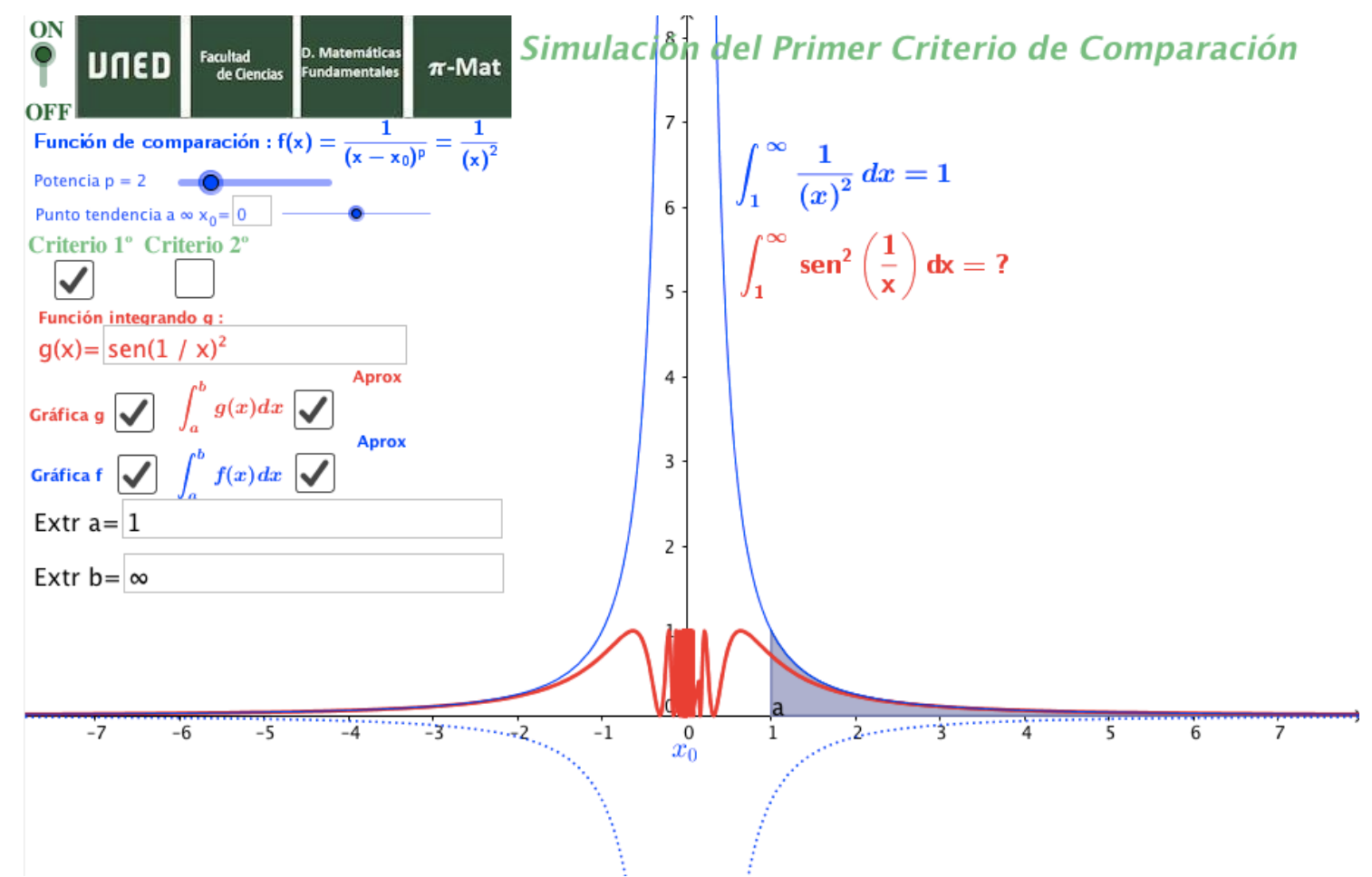

Figura 3: Vista de uso del primer criterio de comparación.

En cierta medida, una vez elegida la función comparación adecuada, se puede tener indicios del carácter de la integral. Pero en muchas ocasiones es necesario una estimación numérica de ese valor. Para este fin, se dispone de una opción de cálculo aproximado (que genera el programa aunque deba ser empleado un método numérico interno) de manera que en cada paso se calcula una integral de Riemann, generándose una secuencia finita de valores que nos presentan sucesivas aproximaciones numéricas.

La opción numérica correspondiente para cada integral impropia está disponible sólo si no está seleccionada la opción integral exacta. En el caso de seleccionar una aproximación numérica para una integral impropia de primera especie, aparece un deslizador para cambiar el extremo superior de la integral de Riemann desde el valor $10^{0}$ hasta el valor $10^{16}$. 
Comunicaciones sobre innovación en la enseñanza y el aprendizaje del Análisis Matemático

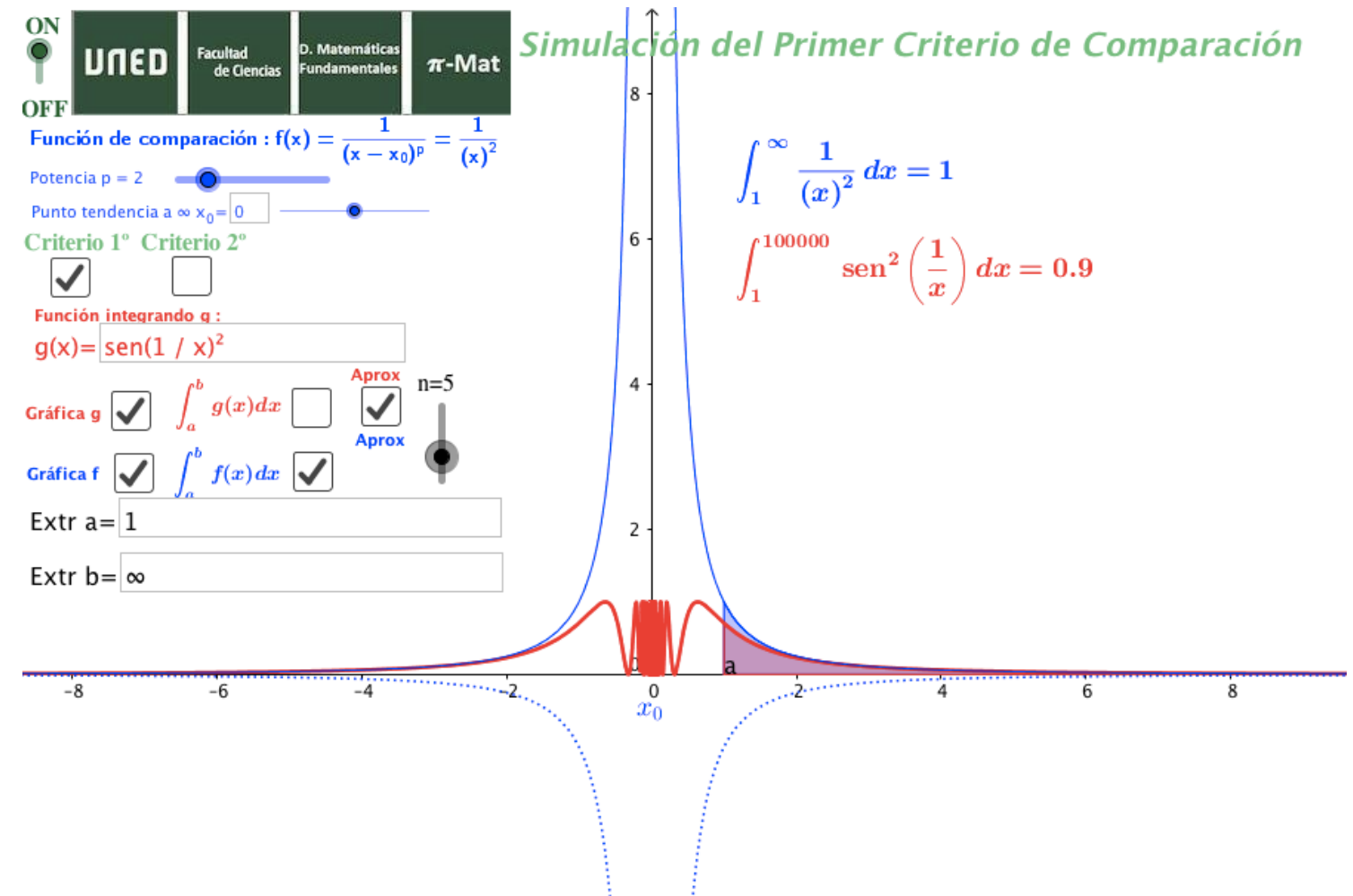

Figura 4: Vista con el cálculo aproximado de la integral impropia de $g(x)$.

Si se opta por la utilización del segundo criterio de comparación, entonces aparece la opción de disponer de la gráfica de la función cociente $\frac{g}{f}$. Al elegir la casilla de selección de esta opción, se presenta la gráfica de esa función cociente y el límite:

$$
\lim _{x \rightarrow \infty} \frac{g(x)}{f(x)}
$$

Al modificar la función comparación podría llegar a la situación de que ese límite es un número no nulo, en ese caso aparece la recta asíntota horizontal de la gráfica de la función cociente y el mensaje de que ambas integrales impropias tienen el mismo carácter. 
Comunicaciones sobre innovación en la enseñanza y el aprendizaje del Análisis Matemático

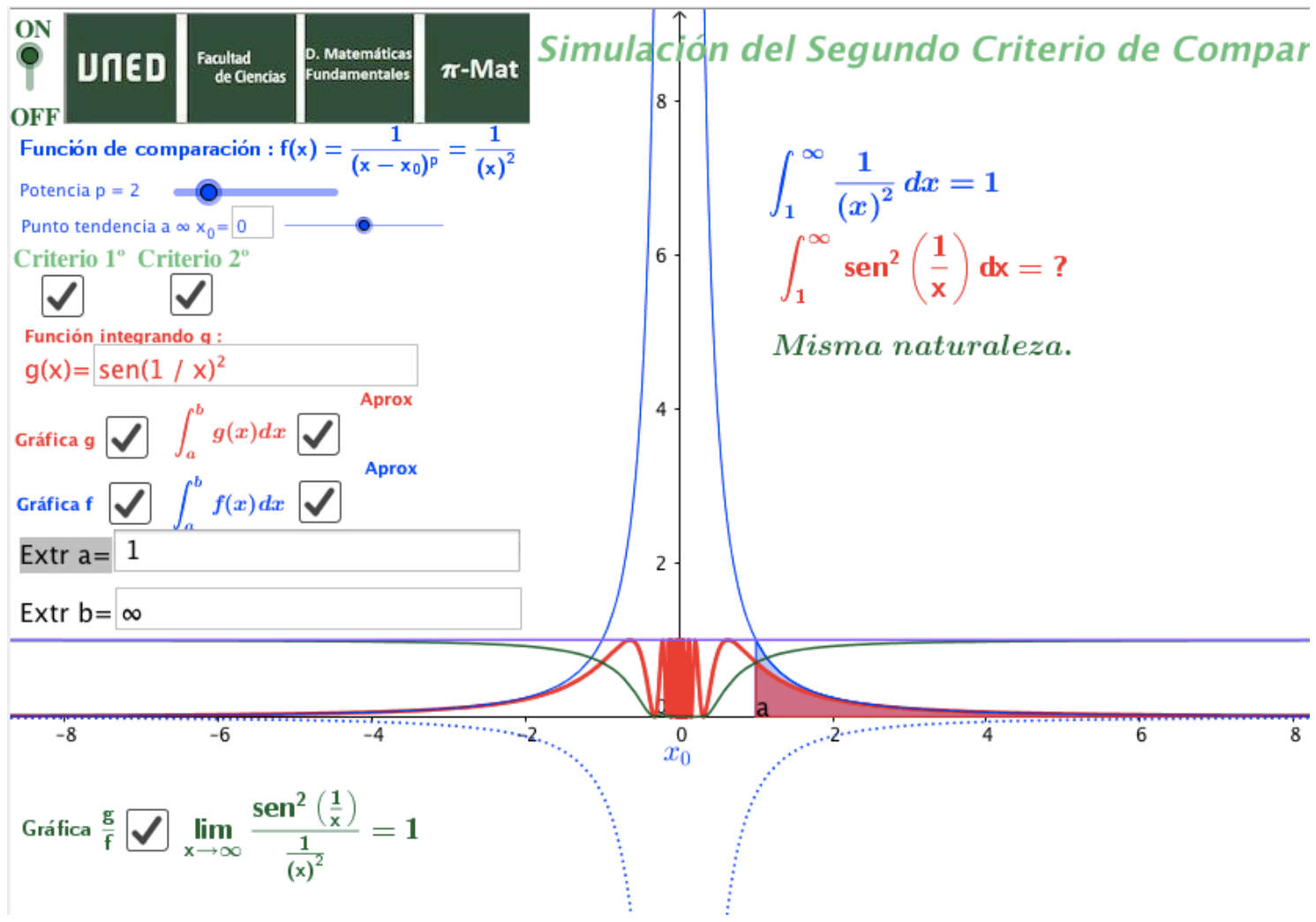

Figura 5: Vista correspondiente al segundo criterio de comparación.

\section{Experiencia educativa}

Dada la naturaleza de nuestra enseñanza, una universidad de educación a distancia, no podemos ofertarle una aplicación abierta totalmente a los estudiantes, puesto que las bondades que se pretenden conseguir con nuestro laboratorio, pudieran ser contrarrestada con alguna actuación libre del estudiante. Con el fin de no generar dificultad didáctica alguna por el mal uso de la aplicación, nos hemos obligado a restringir opciones y experimentos a desarrollar.

En general hemos presentado un listado de experiencias guiadas con un par de funciones integrando para que el estudiante se familiarice con el entorno de simulación experimental. Este es el caso de las integrales impropias

$$
\int_{1}^{+\infty} \operatorname{sen}^{2}\left(\frac{1}{x}\right) d x \text { y } \int_{1}^{+\infty} \frac{\operatorname{sen}^{2}(x)}{x^{2}} d x
$$

que corresponde a funciones integrando positivas. 
Comunicaciones sobre innovación en la enseñanza y el aprendizaje del Análisis Matemático

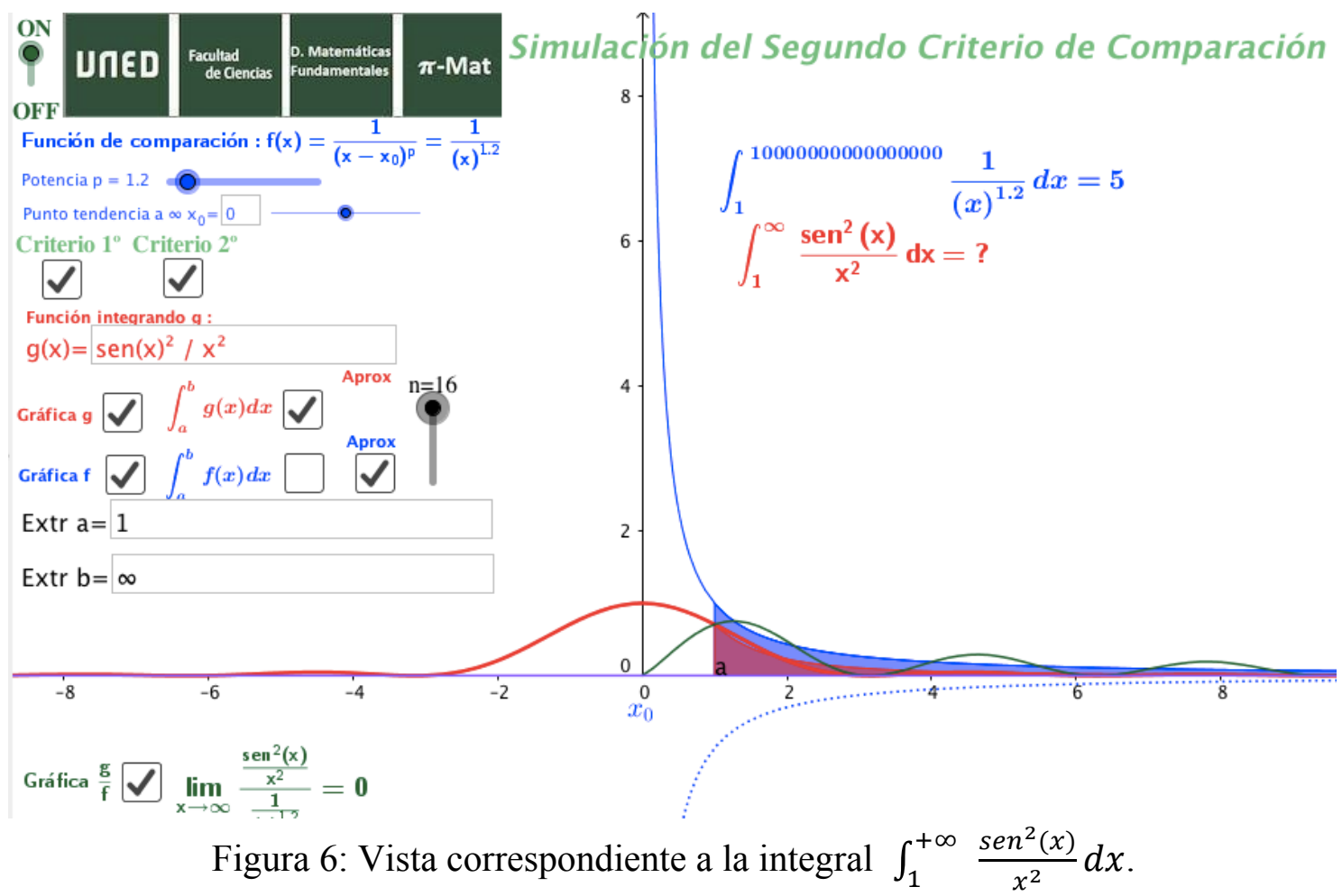

Con posterioridad se afronta otras integrales muy similares a las anteriores con otras funciones integrando con valores positivos y negativos. Por ejemplo,

$$
\int_{1}^{+\infty} \frac{\operatorname{sen}\left(x^{2}\right)}{x^{2}} d x
$$

Posteriormente se aconseja experimentar con las integrales del texto base que el equipo docente recomienda como elemento fuente de conocimiento.

\section{Conclusiones y línea de futuro}

Se ha detectado por comentarios de estudiantes que este laboratorio les ha facilitado la comprensión de esta parte de la materia y para darse cuenta de la dificultad que tiene este tipo de integrales, sobre todo cuando la función integrando no posee una función primitiva conocida.

No disponemos del número de estudiantes que ha utilizado el laboratorio, puesto que tan sólo podemos detectar aquellos que accedieron al laboratorio o a las prácticas recomendadas. Pero no podemos asegurar que estos últimos hayan experimentado.

Entre las futuras líneas de actuación que nos planteamos los siguientes objetivos: 
Comunicaciones sobre innovación en la enseñanza y el aprendizaje del Análisis Matemático

Determinar un método para analizar el impacto del uso de los laboratorios en los procesos de visualización matemática de los estudiantes del Grado de Matemáticas de la UNED.

Desarrollar nuevos materiales didácticos interactivos que integren laboratorios, mini-videos y contenido teórico práctico (GeoGebra permite diseñar libros interactivos).

Extender esta iniciativa a otras asignaturas de Análisis Matemático impartidas por el Departamento de Matemáticas Fundamentales en otras titulaciones (Grado en Física, Grado en Química, Grado en Ciencias Ambientales, etc.)

\section{REFERENCIAS}

1 J. Fernández Novoa. Análisis matemático I. Ed UNED. Madrid (1980).

2 E. Linés Escardó. Análisis matemático. Ed Reverté. Barcelona(1975) 